\title{
Studien über den Milchsäurestoffwechsel beim Ileus.
}

\author{
II. Mitteilung.
}

Milchsäurestoffwechsel in Leber beim Ileus.

Von

Jiro Izumi. $\dagger$

(泉 治 郎)

(Aus der Medizinischen Klinik von Prof. Dr. T. Kato, Tohoku Reichsuniversität zu Sendai.)

Im ersten Bericht ${ }^{12}$ vorliegender Studien habe ieh darauf hingewiesen, dass im Darmverschlussversuch nach erfolgter Unterbindung einzelner Teile des Darmkanals der Milchsäurespiegel des Blutes, obzwar dieser je nach der Höhe der angelegten Ligatur gewisse Unterschiede in der Schnelligkeit und Intensität des Anstiegs zeigt, sich in allen Fällen offensichtlich erhöht. Ferner habe ich auch darauf aufmerksam gemacht, dass diese Milchsäurevermehrung zwar auf den durch das sog. Ileusgift bedingten Störungen verschiedener Organfunktion beruht, vor allem aber mit der der Leber im innigsten und wichtigsten Zusammenhange steht.

Nun wäre es von Interesse und wichtigster Bedeutung, danach zu fahnden, $a b$ und inwieweit beim Mleus der Milchsäurestoffwechsel in der Leber beeinflusst würde. In dieser Hinsicht habe ich die vorliegende Untersuchung angestellt, und zwar in der Absicht, mir Aufklärung darüber zu verschaffen, welche Veränderung beim Ileus die Milchsäureresynthese in der Leber, die an und für sich grosse Mengen von Glykogen enthält, erleiden mag, indem ich, ohne die Leber aus allgemeiner Blutzirkulation ausgeschaltet zu haben, den Milchsäuregehalt des Arterien- und Pfortaderblutes sowie den des Lebervenenblutes zueinander vergleichend bestimmte.

Vers u chs methode:-Als Versuchsmaterial standen 1,8-2,4 kg, im Mittel $2 \mathrm{~kg}$ schwere Kaninchen zur. Verfügung. Jedes Kaninchen, welches man seit dem Abend zuvor nüchtern hielt, wurde auf einem elektrisch erwärmten Tierhalter in der Rückenlage befestigt und

1) I z u mi, Tohokn Journ. Exp. Med., 1933, 22, 201. 
durch subkutane Injektion 25\%iger Urethanlösung im Mengenverhältniss von $4 \mathrm{ccm}$ pro kg Körpergewicht in Narkose gebracht. Die Entnahme der Leberblutproben geschah nach Kato und Kimura, ${ }^{2)}$ indem man Laparotomie durchführte.

Das Verfahren ist nämlich wie folgt: Um das Leberrenenblut zu bekommen zieht man direkt am unteren Teil der Leber ein dünnes Pferdehaar rings um die V. cava inferior heran, stellt damit eine Schlinge her, deren Zug den Blutstrom in der V. cava inf. provisorisch aufhebt. Nun sticht man im subphrenischen Raume die V. cava inf. direkt am oberen Teil der Eintrittstelle der Lebervene ein, wodurch man unter Ausschaltung der Blutströmung aus unteren Extremitäten, beiden Nieren u. s. w. dasjenige Blut, welches nur die Leber durchströmt, gewinnt. Aus der Punktionsstelle kommt nachher ohne besondere Nachbehandlung keine Blutung vor. Zur Entnahme des Pfortaderblutes punktiert man mit einer exakt graduierten, $1 \mathrm{ccm}$ fassenden Spritze, ohne die Blutstauung herbeigeführt zu haben, die Pfortader dicht vor deren Eintritt in die Leber, wobei die Einstichstelle $\mathrm{z}$ wecks Blutstillung sofort nach der Blutentnahme mittels einer kleinen, speziell erdachteten Drahtklemme in möglichst geringer Ausdehnung gefasst wurde. In obiger Weise gelingt es, ohne die Blutströmung in der Pfortader zu verhindern, mehrmalige Punktionen auszuführen. Die arterielle Blutprobe bekommt man ans Carotis, indem man im Voraus in diese eine Kanüle angelegt hat.

Nach der vorbereitenden Operation zur Blutentnahme lässt man das Versuchstier, um etwaigen nachteiligen Einflüssen der Operation vorzubeugen, zwei Stunden lang ausruhen und schritt erst dann zur Blutentnahme. Nachdem man alsdann eine komplete Darmunterbindung in ganz ähnlicher Weise, wie im erster Bericht beschrieben wurde, angelegt hat, verschliesst man die Bauchhöhle durch leichte Naht, befestigt die in die Carotis eingebundene Kanüle mit dem Verband schlaff, entfesselt das Tier und lässt bis zur Frist der zweiten Blutentnahme für 12-24 Stunden beliebige Körperstellung nehmen.

AufGrund des im erster-Bericht angeführten Versuchsergebnisses wurde bei zweiter Blutentnahme das Tier im Zeitpunkt, wo nach der Darmunterbindung Ileussymptome ausgesprochen zutage traten, also beim Verschluss des oberen Darmteils nach 12stündigem Ablauf, beim Verschluss des unteren Teils nach 24 stündigem Ablauf von erster Blutentnahme wiederum auf den Tierhalter gebracht, es wurden dann nach Entfernung der Naht die Laparotomie und die Blutentnahme in derselben Weise, wie vor dem Darmverschluss vorgenommen. Hierbei wurde es nie versäumt, während des obigen Verfahrens das Gedärme mit Tüchern, die in körperwarme Ringer-Lösung eingetaucht worden sind, vor Kälte zu schützen und auch selbstredend übliche aseptische Kautelen streng einzuhalten.

2) Ka to u. Ki mura, Tohoku Journ. Exp. Med., 1933, 21, 298. 
Die Milchsäurebestimmung geschah nach der von Anrep und Cannan ${ }^{3)}$ angegebenen, von Inawashiro und Hayasaka) modifizierten Methode jedesmal an den drei Blutproben: dem arteriellen, dem Lebervenen- und dem Pfortaderblut.

\section{Versuchsergebnis.}

Die an im ganzen 12 Kaninchen vor der Darmunterbindung ermittelten, d. h. normalen Milchsäurewerte des Leberblutes beträgt beim Arterienblut 18,00-26,36 mg/dl, im Mittel 22,34 mg/dl, beim Pfortaderblut 15,43-23,14 mg/dl, im Mittel 19,66 mg/dl, und beim Lebervenenblut 12,86-19,20 mg/dl, im Mittel 16,52 mg/dl; also weist das arterielle Blut den höchsten Milchsäurespiegel auf, dann folgt das Pfortaderblut, während das Lebervenenblut den niedrigsten Wert zeigt.

Dieses Verhältnis des Milchsăurespiegels erweist sich als ganz übereinstimmend mit demjenigen, welches von $\mathrm{Him}$ wich, Kosk of $\mathrm{f}$ und $\left.\mathrm{Nahum},{ }^{5}\right)$ Schneider und Widmann, ${ }^{6)}$ Rose, Giragossintz und Kirstein, ${ }^{7)}$ Harold, Him wich, Koskoff und $\mathrm{Nahum},{ }^{8}$ ) Schneider und Widmann ${ }^{9}$ u. a. angegeben wurde, was auch von Kato und Kimura a an hiesiger Klinik bestätigt worden ist.

Dieser Befund spricht offenbar dafür, dass in der Leber die Milchsäure in das Glykogen umgewandelt ist.

In jüngster Zeit haben Ka to und $\mathrm{Ki} \mathrm{m} \mathrm{u} \mathrm{ra}{ }^{2}$ ) in hiesiger Klinil die Untersuchung angestellt, sich Klarheit darüber zu verschaffen, ob und inwieweit der Milchsäurespiegel des Leberblutes durch wiederholte Blutentnahme beeinflusst würde, und den Nachweis erbracht, dass, obzwar die Blatentnahme Veründerungen in absoluter Höhe des Milchsäurespiegels bewirkt, die Reihenfolge zwischen dem arteriellen, Pfortader- und Lebervenenblute in bezug auf den Milchsäuregehalt immer nach wie vor unbedingt unbeeinflusst bleibt.

\section{Der Milchsäurestoffwechsel in der Leber beim Verschluss am höheren Darmabschnitt (bei Duodenalunterbindung).}

Nach dem Versuchsergebnisse in meinem ersten Bericht ${ }^{1)}$ und dem von $\mathrm{Odaira},{ }^{10)} \mathrm{Ch} \mathrm{o}{ }^{11)} \mathrm{u}$. a. ist es verständlich, dass beim Tier mit 6-12

\footnotetext{
3) Anrep u. Cannan, Journ. Physiol., 1923, 58, 244.

4) In awashiro u. Hayasaka, Tohoku Journ. Exp. Med., 1928, 12, 1.

5) Hinwich, Kosk off u. Na hu m, Proc. Soc. Exp. Biol. and Med., 1928, 25, 347.

6) Schneider u. Widmann, Kl. Wochenschr., 1928, 646.

7) Rose, Giragossintz n. Kirstein, Proc. Soc. Exp. Biol. and Med., 1930, 27, 523.

8) Harold, Himwich, K osk of u. Nahum, Journ. Biol. Chem., 1930, 85, 571.

9) Schneider u. Widmann, Kl. Wochenschr., 1930, 761.

10) Od a ir a, Tohoku Journ. Exp. Med., 1922, 2, 570.

11) C ho, Chosen Igaku Zasshi, 1930, 20, 1918.
} 
Stunden anhaltender Duodenalunterbindung bereits Azidose, Leberautolyse und andere Symptome auftreten, dementsprechend auch die Blutmilchsäure erheblich zunimmt. Aus diesem Grund habe ich absichtlich in 12stündigem Ablanf nach Anlegen der Ligatur das Arterienblut sowie das Pfortader- und Lebervenenblut entnommen und den jeweiligen Milchsäuregehalt bestimmt, um dann die Milchsäurewerte mit den vor Unterbindung ermittelten Milchsäurewerten miteinander zu vergleichen.

Die Feststellung, dass der Milchsäuregehalt des arteriellen Blutes 12 Stunden nach Unterbindung beträchtlich vermehrt war, stimmt mit dem Ergebnisse im ersten Bericht überein. Der Milchsäurespiegel des Pfortaderblutes erhöhte sich auch in allen Fällen ausnahmslos erheblich, und dieser Anstieg war gewissermassen im Grade mehr oder minder grösser als dieselbe des arteriellen Blutes. Im Lebervenenblute wies der Milchsäuregehalt in allen Fällen die allergrösste Zunahme auf, welche am Grad dieselbe der beiden vorerwähnten Blutarten weitaus übertraf (Tab. 1 u. Fig. 1).

Tabelle 1.

Die Veränderungen des Milchsäurespiegels der Leberblute beim Verschluss am Ende des Duodenums.

\begin{tabular}{|c|c|c|c|c|c|}
\hline \multirow{2}{*}{ Nummer } & \multirow{2}{*}{$\begin{array}{c}\text { Körperge- } \\
\text { wicht (kg) u. } \\
\text { Geschlecht }\end{array}$} & \multirow{2}{*}{ Blut } & \multirow{2}{*}{$\begin{array}{l}\text { Vor d. } \\
\text { Verschluss } \\
(\mathrm{mg} / \mathrm{dl})\end{array}$} & \multicolumn{2}{|c|}{12 Stdn. nach d. Verschluss } \\
\hline & & & & $\mathrm{mg} / \mathrm{dl}$ & Diff. in $\%$ \\
\hline 1 & 2,15 우 & $\begin{array}{l}\text { Arterie } \\
\text { V. portae } \\
\text { V. hepatica }\end{array}$ & $\begin{array}{l}23,79 \\
20,57 \\
16,71\end{array}$ & $\begin{array}{l}33,43 \\
32,14 \\
34,71\end{array}$ & $\begin{array}{r}+40,5 \\
+\quad 56,3 \\
+112,5\end{array}$ \\
\hline 2 & 2,35 우 & $\begin{array}{l}\text { Arterie } \\
\text { V. portae } \\
\text { V. hepatica }\end{array}$ & $\begin{array}{l}21,85 \\
18,00 \\
14,79\end{array}$ & $\begin{array}{l}36,64 \\
35,36 \\
39,21\end{array}$ & $\begin{array}{r}+67,7 \\
+96,4 \\
+165,1\end{array}$ \\
\hline 3 & 1,98 ㅇ & $\begin{array}{l}\text { Arterie } \\
\text { V. portae } \\
\text { V. hepatica }\end{array}$ & $\begin{array}{l}25,07 \\
22,50 \\
19,29\end{array}$ & $\begin{array}{l}41,79 \\
39,86 \\
47,57\end{array}$ & $\begin{array}{r}+66,7 \\
+\quad 77,2 \\
+146,6\end{array}$ \\
\hline 4 & 2,05 우 & $\begin{array}{l}\text { Arterie } \\
\text { V. portae } \\
\text { V. hepatica }\end{array}$ & $\begin{array}{l}18,00 \\
15,43 \\
12,86\end{array}$ & $\begin{array}{l}39,86 \\
37,29 \\
46,93\end{array}$ & $\begin{array}{r}+121,4 \\
+141,7 \\
+264,9\end{array}$ \\
\hline 5 & 2,40 ' & $\begin{array}{l}\text { Arterie } \\
\text { V. portae } \\
\text { V. hepatica }\end{array}$ & $\begin{array}{l}23,14 \\
20,57 \\
16,71\end{array}$ & $\begin{array}{l}40,50 \\
38,57 \\
48,21\end{array}$ & $\begin{array}{r}+50,7 \\
+\quad 87,5 \\
+196,9\end{array}$ \\
\hline 6 & $2,03 \curvearrowright$ & $\begin{array}{l}\text { Arterie } \\
\text { V. portae } \\
\text { V. hepatica }\end{array}$ & $\begin{array}{l}20,57 \\
18,00 \\
15,43\end{array}$ & $\begin{array}{l}36,64 \\
35,36 \\
41,79\end{array}$ & $\begin{array}{r}+78,1 \\
+\quad 96,4 \\
+170,8\end{array}$ \\
\hline & urchschnitt & $\begin{array}{l}\text { Arterie } \\
\text { V. portae } \\
\text { V. hepatica }\end{array}$ & $\begin{array}{l}22,07 \\
19,18 \\
15,79\end{array}$ & $\begin{array}{l}38,14 \\
36,46 \\
43,07\end{array}$ & $\begin{array}{r}+\quad 70,9 \\
+\quad 92,6 \\
+176,1\end{array}$ \\
\hline
\end{tabular}




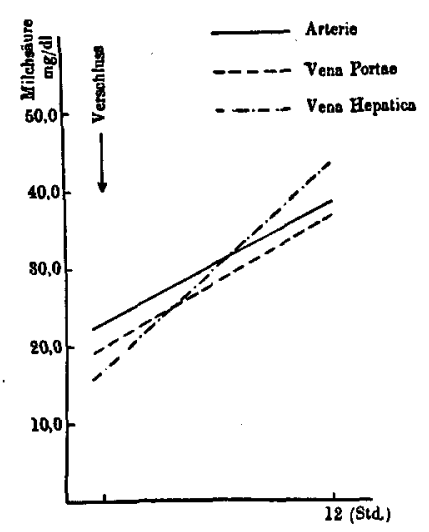

Fig. 1. Milchsäuregehalt der Leberblute beim Duodenumverschluss. (Durchsehnitts wert)

Die Vermehrung des Milchsäuregehaltes, auf den prozentischen Gehalt umgerechnet, zeigt folgende Verhältnisse : Im arteriellen Blut beträgt er $40,5-121,4 \%$, im Mittel $70,9 \%$, im Pfortaderblut 56,3-141,7\%, im Mittel $92,6 \%$, und im Lebervenenblut $112,5 \%$ $-264,9 \%$, im Mittel $176,1 \%$.

Ein Überblick über obige Zahlenwerte lehrt uns, dass nach erfolgter Unterbindung sich der Milchsäurewert des Pfortaderblutes stärker als derselbe des arteriellen Blutes vermehrt und nach 12stündigem Ablauf an ihn heranzutreten geneigt ist, dass der Milchsäurewert des Lebervenenblutes eine recht erhebliche Zunahme, welche am Grad Werte der übrigen beiden Blutarten weitaus übertrifft, aufweist, so dass die vor Unterbindung gefundene Reihenfolge der Milchsäurewerte in 3 Blutarten eine Umwandelung erfährt; der Milchsäurewert des Lebervenenblutes nimmt nämlich nunmehr die höchste Stellung ein, bei weitem herabfallend folgt ihm der Milchsäurewerte des arteriellen Blutes und nächst demselben kommt der Milchsäurewert des Pfortaderblutes in etwas herabgesetztem Masse.

Dieses Ergebnis spricht offenbar dafür, dass der Vorgang der Milchsäureresynthese in der Leber durch Leberläsionen gestört ist und aus der Tatsache, dass der Milchsäuregehalt des Lebervenenblutes in weitaus stärkerem Grad als im Pfortaderblut vermehrt ist, dürfte man wohl darauf schliessen, dass der glykogenolytische Vorgang in der Leber durch das sog. Tleusgift beschleunigt wird und als ein intermediäres Spaltprodukt die Milchsäure vermehrt auftritt. Dies kann auch durch den Nachweis von Seulberger, ${ }^{12)}$ Takemur'a ${ }^{13)}$ u. a., dass beim Ileus ein auffallender Schwund von Leberglykogen stattfindet, gestützt werden.

\section{Der Milchsäurest offwechsel in der Leber beim Verschluss am unteren Darmabschnitt (bei Ileumunterbindung).}

Von demselben Gesichtspunkt, wie im vorangegangenen er'wäht wurde, ausgehend, wurde in 24 Stunden nach erfolgter Darmunter-

12) Seulberger, Bruns' Beitr. z. kl. Chir., 1929, 148, 583.

13) Takemura, Tokyo Igakkai Zasshi, 1931, 45, 1459. 
bindung das Blut aus der Arterie, Pfortader und Lebervene entnommen, den Milchsäuregehalt in jeder Blutprobe bestimmt und diese Werte mit den vor Unterbindung gewonnenen Werte verglichen.

Wenn auch nicht so bedeutend wie bei der Duodenumunterbindung erhöht sich doch hierbei der Milchsäurespiegel des arteriellen Blutes 24 Stunden nach Unterbindung eben so deutlich, wie im ersten Bericht gezeigt wurde. Der des Pfortaderblutes nimmt auch ausnahmslos deutlich $z \mathfrak{u}$, und zwar in ziemlich grösserem Masse als derselbe des arteriellen Blutes. Im Lebervenenblut zeigt er in allen Fällen ohne Ausnahme eine beträchtliche Zunahme, welche am Grad dieselbe in beiden erwähnten Blutsorten übertrifft (Tab. 2 u. Fig. 2).

\section{Tabelle 2.}

Die Veränderungen des Milchsäurespiegels der Leberblute beim Verschluss am Ende des Ileum.

\begin{tabular}{|c|c|c|c|c|c|}
\hline \multirow{2}{*}{ Nummer } & \multirow{2}{*}{$\begin{array}{c}\text { Körperge- } \\
\text { wicht (kg) u. } \\
\text { Geschlecht }\end{array}$} & \multirow{2}{*}{ Blut } & \multirow{2}{*}{$\begin{array}{l}\text { Vor d. } \\
\text { Verschluss } \\
\text { (mg/dl) }\end{array}$} & \multicolumn{2}{|c|}{24 Stdn. nach d. Verschluss } \\
\hline & & & & $\mathrm{mg} / \mathrm{dl}$ & Diff. in $\%$ \\
\hline 1 & 2,06 우 & $\begin{array}{l}\text { Arterie } \\
\text { V. portae } \\
\text { V. hepatica }\end{array}$ & $\begin{array}{l}22,50 \\
19,93 \\
16,71\end{array}$ & $\begin{array}{l}25,71 \\
23,79 \\
24,43\end{array}$ & $\begin{array}{l}+14,3 \\
+19,4 \\
+46,2\end{array}$ \\
\hline 2 & $2,00 \hat{\delta}$ & $\begin{array}{l}\text { Arterie } \\
\text { V. portae } \\
\text { V. hepatica }\end{array}$ & $\begin{array}{l}21,21 \\
18,00 \\
14,79\end{array}$ & $\begin{array}{l}26,36 \\
25,07 \\
28,29\end{array}$ & $\begin{array}{r}+19,6 \\
+39,3 \\
+91,3\end{array}$ \\
\hline 3 & 1,98 상 & $\begin{array}{l}\text { Arterie } \\
\text { V. portae } \\
\text { V. hepatica }\end{array}$ & $\begin{array}{l}20,57 \\
19,29 \\
18,64\end{array}$ & $\begin{array}{l}27,00 \\
26,36 \\
28,29\end{array}$ & $\begin{array}{r}+31,3 \\
+36,7 \\
+51,8\end{array}$ \\
\hline 4 & 2,08 우 & $\begin{array}{l}\text { Arterie } \\
\text { V. portae } \\
\text { V. hepatica }\end{array}$ & $\begin{array}{l}24,43 \\
21,85 \\
18,64\end{array}$ & $\begin{array}{l}29,57 \\
28,29 \\
29,57\end{array}$ & $\begin{array}{r}+21,0 \\
+29,5 \\
+58,6\end{array}$ \\
\hline 5 & 2,17 oิ & $\begin{array}{l}\text { Arterie } \\
\text { V. portae } \\
\text { V. hepatica }\end{array}$ & $\begin{array}{l}26,36 \\
23,14 \\
19,29\end{array}$ & $\begin{array}{l}29,57 \\
28,93 \\
28,93\end{array}$ & $\begin{array}{r}+12,2 \\
+25,0 \\
+49,9\end{array}$ \\
\hline 6 & 2,25 \& & $\begin{array}{l}\text { Arterie } \\
\text { V. portae } \\
\text { V. hepatica }\end{array}$ & $\begin{array}{l}20,57 \\
18,64 \\
15,43\end{array}$ & $\begin{array}{l}24,43 \\
23,79 \\
25,07\end{array}$ & $\begin{array}{r}+18,8 \\
+27,6 \\
+62,5\end{array}$ \\
\hline & urchschnitt & $\begin{array}{l}\text { Arterie } \\
\text { V. portae } \\
\text { V. hepatica }\end{array}$ & $\begin{array}{l}22,61 \\
20,14 \\
17,25\end{array}$ & $\begin{array}{l}27,11 \\
26,04 \\
27,43\end{array}$ & $\begin{array}{r}+19,5 \\
+29,6 \\
+60,1\end{array}$ \\
\hline
\end{tabular}

Rechnet man die Zunahme des Milchsäuregehaltes auf prozentuale Zahlen um, so bekommt man folgende Ziffern : Beim arteriellen Blut 12,2-31,3\%, im Mittel 19,5\%, beim Pfortaderblut 19,4-39,3\%, im Mittel 29,6\% und beim Lebervenenblut 46,2-91,3\%, im Mittel 60,1\%. 


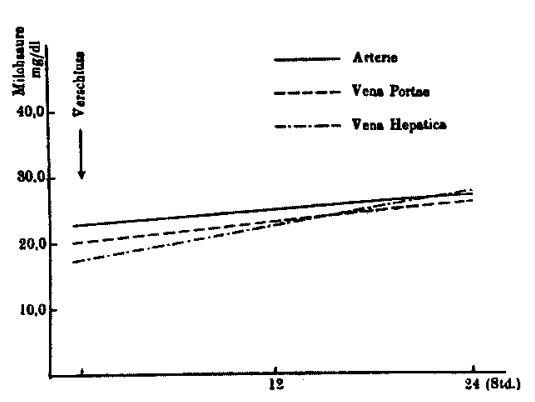

Fig. 2. Milchsäuregehalt der Leberblute beim lleumverschluss, (Durchschnittswert)

Aus oben geschilderten Zahlenangaben geht hervor, dass, obwohl nach dem Darmverschluss der Milchsäuregehalt des Pfortaderblutes sehr stärker als derselbe des Arterienblutes zunimmt und in 24stündigem Ablauf an denselben herantritt, dennoch er demselben untersteht, und dass der Milchsäuregehalt des Lebervenenblutes eine recht ausgesprochene Zunahme aufweist und im Grad der Zunalıme die Milchsäurewerte der übrigen beiden Blutsorten weitaus überwiegt. Wird der Milchsäurespiegel des Lebervenenblutes in einzelnen Fällen betrachtet, so bemerkt man, dass in Fall 1 und 5 er dem des Arterienblutes näher rückt, doch demselben nachsteht, dass in Fall 4 er denselben Wert wie der Wert des Arterienblutes erreicht, während er in übrigen Fällen sogar diesen einigermassen übertrifft. Auch auf den Durchschnittswert bezogen, übertrifft der Milchsäurewert des Lebervenenblutes denselben des Arterienblutes ein wenig und steht naturgemäss höher als der des Pfortaderblutes.

Kurzum verhält sich der Blutmilchsäurespiegel auch bei der Unterbindung des unteren Ileums in qualitativ ähnlicher, wenn auch nicht so bedeutender Weise, wie bei der Unterbindung am unteren Duodenum, indem die vor Unterbindung ermittelte Reihenfolge bezüglich des Milchsäuregehaltes, wie das Arterienblut > das Pfortaderblut $>$ das Lebervenenblut, in 24stündigem Ablauf nach Unterbindung. in ganz umgekehrter Weise in die Reihenfolge wie das Lebervenenblut $>$ das Arterienblut $>$ das Pfortaderblut umgewandelt ist. Demnach muss angenommen werden, dass es sich hier, wiederum in ähnlicher, wenn auch nicht so intensiver Weise, wie bei der Duodenalunterbindung, un durch Leberläsionen bedingte Störungen des Vorgangs der Milchsäureresynthese in der Leber handelt, und dass andererseits auch im Anschluss an den durch Giftwirkung verursachten Leberglykogenschwund vermehrte Milchsäurebildung vor sich geht.

\section{Kritische Besprechung.}

Es ist eine allbekannte Tatsache, dass die Leber an den Entgiftung'sprozessen gegen alle Gifte beteiligt ist und ein Zentralorgan für 
den allgemeinen Stoffwechsel darstellt; vor allem aber für den Kohlehydratstoffwechsel, demgemäss auch für den Milchsäurestoffwechsel spielt sie die Hauptrolle. Dies ist schon früher von $E m b d e n$ und Kraus ${ }^{14)}$ Embden sowie Is a a ${ }^{15)}$ und Isaa ${ }^{16)}$ hervorgehoben worden. Und aus dem Umstand, dass die Milchsäure in dem aus der Leber abfliessenden Lebervenenblut weniger als in dem der Leber zuströmenden arteriellen und Pfortaderblut enthalten ist, können wir, wie bereits angeführt wurde, mit Sicherheit darauf schliessen, dass in der Leber der Vorgang der Milchsäureresynthese lebhaft stattfindet. Dieser wird bei gestörter Leberfunktion notwendigerweise in Mitleidenschaft gezogen.

Nach Adler und Lange ${ }^{17}$ ) haben Steigerung des Milchsäurespiegels bei Leberkranken konstatiert und verzögerte Milchsiüureresynthese bei der Lebererkrankung angenommen. Derra und Ernst ${ }^{18}$ geben an, dass bei akuter Leberatrophie Milchsäure im Blute und Aminosäuren im Harn in erheblichem Masse zunehmen und Beckmann, ${ }^{19}$ Beckmann und Mirsalis, ${ }^{20}$ Hochrein und Meier ${ }^{21)}$ n. a. haben bei der Leberschädigung eine deutliche Steigerung des

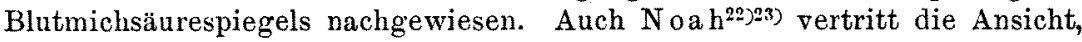
dass bei den durch Phosphorvergiftung und andere Ursachen hervorgerufenen Leberfunktionsstörungen die Blutmilehsäure zunimmt. Weiterhin hat Mizu$n \mathrm{o}^{2+325)}$ durch experimentelle Untersuchung festgestellt, dass bei Störungen der Leberfunktion, gleichviel, durch welche Ursachen sie auch herbeigeführt werden mögen, stets nebst vermehrtem Milchsäuregehalt im Blut auch die gesteigerte Bildung der Milchsäure in der Galle vonstatten geht und dass die Schwankungen der Blutmilchsäure von intrahepatischen Parenchymzellen abhängig sind.

Nun unterliegt es keinem Zweifel, dass beim Auftreten der Ileussymptome die Leber die wichtigste Bedeutung hat, was auch aus der Tatsache, dass hierbei das sog. resorbierte Ileusgift zuerst in der Leber, die als Filter der Gifte wirkt, sich ansammelt, ohne weiteres anerkannt werden dürfte.

Werelius, ${ }^{2(6)}$ Seulberger ${ }^{19)}$ Takemura ${ }^{13)}$ u. a. legen grosses Gewicht auf die bei Ileus eintretenden Funktionsstörungen der Leber und wollen die hierdurch hervorgerufene Stoff wechselstörung als Todesursache beschuldigt wissen.

14) Em bden u. Kra u s, Biochem. Ztschr, 1912, 45, 1.

15) Embden u. Is a ac, Ho p pe-Seyler's Ztschr., 1917, 99, 297.

16) Is a a c, Berl. kl. Wochenschr., 1919, 940.

17) A d ler u. L ange, Deut. Arch. f. kl. Med., 1927, 157, 129.

18) Derra u. Ernst, Ztschr. f. d. ges. exp. Med., 1927, 57, 657.

19) $\mathrm{Beckm}$ a n n, Kl. Wochensehr., 1929, 2229.

20) Beckmanu u. Mirsalis, Deut. Arch. f. kl. Med., 1928, 159, 129.

21) Hochrein u. Meier, Deut. Arch. f. kl. Med., 1928, 161, 59.

22) Noah, Kl. Wochensehr., 1927, 1465.

23) D o, Ztschr. f. d. ges. exp. Med., 1928, 61, 59.

24) Mizu u o, Jikken Shokakibyogaku, 1929, 4, 571 u. 581 .

25) D o, Jap. Journ. Gastroenterol., 1931, 3, 175.

26) Wereliu s, Jour'n. Americ. Med. Assoc., 1922, 79, 535. 
Cho ${ }^{11)}$ richtet sein Hauptaugenmerk auf die Leberautolyse. Shibata ${ }^{27}$ hat durch pathologisch-histologische Untersuchung beim Ileum den Beweis dafür geliefert, dass hierbei vor allen Dingen die parenchymatöse Degeneration der Leber sich geltend macht und dieselbe von allen betroffenen Organen das aufs rascheste und intensivste befallene Organ ist.

Wie aus obigen Angaben von verschiedenen Autoren klar hervorgeht, erleidet beim Ileus die Leber sowohl in funktioneller wie auch organischer Hinsicht die intensivste Schädigung und es versteht sich von selbst, dass alle in der Leber sich abspielenden Stoffwechselvorgänge im stärksten Grad beeinträchtigt, namentlich der Kohlehydratstoffwechsel und dementsprechend auch der intrahepatischeMilchsäurestoffwechsel in erheblichem Masse gestört werden müssen.

Die auffallendste Vermehrung des Milchsäuregehaltes des Lebervenenblutes, die in vorliegender Arbeit nach Darmunterbindung auftrat, und welche die hierbei ebenfalls erhobene Zunahme des Milchsäuregehaltes des arteriellen und Pfortaderblutes bei weitem übertraf, liefert zweifellos den Beweis dafür, dass nicht allein der Vorgang der Milchsäureresynthese in der Leber behindert ist, sondern auch infolge der durch Ileusgift verur'sachten geförderten Glykogenolyse die vermehrte Milchsäurebildung vonstatten geht.

In den beiden Versuchen, nämlich beim Verschluss sowohl am unteren als auch am oberen Teil des Darmrohrs wurde die Milchsäure $\operatorname{der} \mathrm{V}$. portae mehr als dieselbe des Arterienblutes vermehrt gefunden; dies ist darauf zuruickzuführen, dass in den im Pfortadersystem gelegenen Organen und Geweben, im besonderen aber in glatter Muskulatur u. s. w. die Milchsäurebildung in vermehrtem Masse vor sich geht, bzw. die Milchsäureresynthese durch Ileusgift gestört ist.

\section{Zusammenfassung.}

Um die Veränderungen der Milchsäureresynthese in Leber beim Darmverschluss zu verfolgen, wurde der Milchsäuregehalt des Arterien-, des Pfortader- und des Lebervenenblutes vergleichend bestimmt, woraus sich folgendes ergab:

1. Beim Duodenalverschluss wurde im 12stündigem Ablauf nach Unterbindung der Milchsäuregehalt des arteriellen, Pfortader-sowie des Lebervenenblutes bestimint und diese Werte mit den einzelnen, vor Unterbindung ermittelten Werten verglichen; es hat sich hierbei herausgestellt, dass der Milchsäuregehalt in jedem Blut eine ausgesprochene Zunahme aufwies, und zwar der Grad dieser Zunahme im Arterienblut sich als relativ bescheidend, im Pfortaderblut als riem-

27) Shibata, Keio Igaku, 1931, 11, 2645. 
lich grösser und im Lebervenenblut entschieden als überwiegend erwies, so dass die vor Unterbindung erhobene Reihenfolge, wie das Arterienblut $>$ das Pfortaderblut $>$ das Lebervenenblut, nunmehr umgekehrterweise in die Reihenfolge wie das Lebervenenblut>das Arterienblut $>$ das Pfortaderblut ungewandelt war.

2. Beim Ileumverschluss verhält sich der Milchsäurespiegel der drei Blutproben im 24stündigem Ablauf nach Unterbindung auch in ähnlicher wenn auch nicht so bedeutender Weise wie beim Duodenalver'schluss.

3. Die auffallendste Vermehrung des Milchsäuregehaltes des Lebervenenblutes deutet darauf hin, dass beim Ileus die Milchsäureresynthese in der Leber erheblich gestört ist, dass ferner noch dabei gesteigerter Glykogenschwund stattfindet, der zu vermehrter Milchsäurebildung führt. Auch die Feststellung, dass das Verhältnis der Erhöhung des Milchsäurespiegels im Pfortaderblut grösser als im Arterienblut ist, dürfte wohl darauf beruhen, dass nach erfolgtem Darmverschluss der Milchsäurestoffwechsel, der sich in den im Pfortadersystem gelegenen Organen abspielt, auch mehr oder weniger gestört ist. 